Brain size in autism. Cerebral cortical enlargement is reported in children with ASD examined by MRI at both 2 and 4-5 years of age (Hazlett HC et al. Arch Gen Psychiatry May 2011;68(5):467-476). A disproportionate enlargement in temporal lobe white matter was observed in ASD children $(\mathrm{n}=38) \mathrm{cf}$ controls $(\mathrm{n}=21)$. No significant difference from controls was observed in the rate of brain growth between 2 and 4 years of age. Brain enlargement observed in children with ASD is a consequence of an increased rate of brain growth with onset postnatally, before age 2 years and probably in the latter part of the first year of life. This early brain enlargement with increased cortical volume, but not cortical thickness, may be associated with increased cortical surface area and a distinct pathogenic mechanism. The early cortical overgrowth in young children with ASD is followed by a period of cortical thinning during adolescence and adulthood. (Wallace GL et al. Brain 2010;133:3745-3754).

\title{
ARACHNOID CYSTS CAUSING SYMPTOMATIC INCREASED INTRACRANIAL PRESSURE
}

Four children, ages 12 to 15 years, with intracranial arachnoid cysts complicated by increased intracranial pressure are reported from Children's Hospital of Philadelphia, PA. They presented with recent onset or increasing headaches, exacerbated by the supine position. Visual acuity was normal. Optic discs were swollen. Opening pressures at LP were 37 to $54 \mathrm{~cm} \mathrm{H} 2 \mathrm{O}$. All failed to respond to conservative medical therapies, but symptoms improved after a surgical shunting procedure. The arachnoid cyst was in the left middle temporal fossa and was complicated by a Chiari I malformation in 3 patients; one patient had hydrocephalus diagnosed at 18 months and a posterior fossa cyst. Three patients were previously treated with a cystoperitoneal shunt before age 2 and required shunt revision. (Prasad S, Avery RA, Campomanes A, Sutton LN, Liu GT. Symptomatic increased intracranial pressure due to arachnoid cysts. Pediatr Neurol May 2011;44:377380). (Respond: Dr Prasad, Division of Neuro-Ophthalmology, Brigham and Women's Hospital, 75 Francis St, Boston, MA 02115. E-mail: sprasad2@partners.org).

COMMENT. Usually benign, arachnoid cysts are rarely complicated by increased intracranial pressure that may require a surgical shunt procedure. The association of a Chiari I malformation with an arachnoid cyst in 3 of these patients is noteworthy, but a possible relation to the headaches and increased intracranial pressure is undetermined. Other symptoms sometimes associated with arachnoid cyst include attention deficit hyperactivity disorder, not alluded to in this report.

\section{OUTCOMES OF BILATERAL STURGE-WEBER SYNDROME}

Researchers at Wayne State University School of Medicine, Detroit, MI studied 14 children with bilateral Sturge-Weber syndrome, representing $12.7 \%$ of a total of 110 patients with the disorder. Glucose positron emission tomography had an asymmetric pattern, one hemisphere was hypometabolic in 3 patients. Signs of cerebral atrophy or vascular abnormalities on MRI were more pronounced on one side. Long-term follow-up data available for 8 of the 14 patients covered 1-16 years (median, 9 years). Age at onset of seizures in 14 patients ranged from 2 weeks to 7.1 years (median, 6 months). Seizure 
type was mostly complex partial, and seizure control was satisfactory in $6(47 \%)$. Patients with poor seizure control manifested frontal glucose hypometabolism on PET. Nine patients $(64 \%)$ had hemiparesis, with contralateral frontal hypometabolism. Bilateral frontal and temporal hypometabolism was coupled with severe developmental impairment. No patient with good seizure control had severe developmental impairment. Developmental impairment was not related to age at onset of first seizure. Autism in 2 patients was associated with bitemporal hypometabolism on PET. Three patients who underwent epilepsy surgery had complete remission of refractory seizures but development was only partially improved. With or without resective surgery, good seizure control is achieved in about $50 \%$ of patients with bilateral Sturge-Weber syndrome. (Alkonyi B, Chugani HT, Karia S, Behen ME, Juhasz C. Clinical outcomes in bilateral Sturge-Weber syndrome. Pediatr Neurol June 2011;44:443-449). (Respond: Dr Juhasz, Dept of Pediatrics, Wayne State University School of Medicine, 3901 Beaubien Blvd, Detroit, MI 48201. E-mail: juhasz@pet.wayne.edu).

COMMENT. PET studies are important in the evaluation of children with bilateral Sturge-Weber syndrome as follows: 1) prediction of outcome, seizure control, and impairment of development and cognition; 2) risk of autism related to bitemporal hypometabolism; and 3) determination of functional integrity of the nonresected hemisphere before surgery. Early resection of a hemisphere or focus may be beneficial in some bilateral cases with intractable seizures. Persistent seizures may contribute to neurocognitive decline.

\section{METABOLIC AND DEGENERATIVE DISORDERS}

\section{CEREBRAL FOLATE DEFICIENCY SYNDROMES}

Neurologists and geneticists at the University of Barcelona, Spain and other centers analyzed cerebral folate levels in 584 children with neurologic disorders that required diagnostic lumbar puncture. Deficiency of 5-methyltetrahydrofolate (5-MTHF) was detected in the CSF of $71(12 \%)$ patients. Mild to moderate deficiency $(n=63 ; 19-63$ $\mathrm{nmol} / \mathrm{L}$ ) was associated with perinatal asphyxia, CNS infection, and diseases of probable genetic origin (inborn errors of metabolism, white matter disorders, Rett syndrome, 'or epileptic encephalopathies). Severe 5-MTHF depletion $(n=8$; range, $0.6-13 \mathrm{n} / \mathrm{mol} / \mathrm{L})$ was detected in specific mitochondrial disorders, metabolic defects, or cerebral degenerations (severe MTHF reductase deficiency, Kearns-Sayre syndrome, biotin-responsive striatal necrosis, acute necrotizing encephalitis, and FOLRI transporter gene defect). CSF folate levels correlated with plasma levels in cerebral folate deficiency. Thirty-five of 71 patients (49\%) with decreased 5-MTHF had other abnormalities of biogenic amines, homovanillic acid, 5-hydroxyindoleacetic acid, and pterins in CSF. (Perez-Duenas B, Ormazabal A, Toma C, et al. Cerebral folate deficiency syndromes in childhood. Clinical, analytical, and etiologic aspects. Arch Neurol May 2011;68(5):614-621). (Respond: Belen Perez-Duenas MD PhD, Dept of Neurology, Hospital Sant Joan de Deu, 08950 Esplugues, Barcelona, Spain. E-mail: bperez(ahsjdbcn.org). 